\title{
The early warning of aquatic organophosphorus pesticide contamination by on-line monitoring behavioral changes of Daphnia magna
}

\author{
Zongming Ren $\cdot$ Jinmiao Zha $\cdot$ Mei Ma $\cdot$ \\ Zijian Wang $\cdot$ Almut Gerhardt
}

Received: 24 August 2006 / Accepted: 22 January 2007 / Published online: 10 February 2007

(C) Springer Science + Business Media B.V. 2007

\begin{abstract}
In this paper, the movement behavior of Daphnia magna was studied as a bio-indicator of organophosphorous pesticide (OP) contamination, using an on-line bio-monitoring method, the Multispecies Freshwater Biomonitor ${ }^{\circledR}$. A static test of acute toxicity test revealed the $24-\mathrm{h}$ and $48-\mathrm{h} \mathrm{LC}_{50}$ values (95\% confidence limit) for Daphnia magna to be respectively $0.45 \mu \mathrm{g} / \mathrm{l}$ and $0.21 \mu \mathrm{g} / \mathrm{l}$ for dipterex $3.80 \mu \mathrm{g} / \mathrm{l}$ and $0.90 \mu \mathrm{g} / \mathrm{l}$ for malathion, and $1.25 \mu \mathrm{g} / \mathrm{l}$ and $0.38 \mu \mathrm{g} / \mathrm{l}$ for parathion. The behavior strength of Daphnia magna was a sensitive indicator of sublethal OP stress and resulted in significant concentrationresponse relationships for the three OPs. Increasing OP concentration will result in more intensive behavioral responses of Daphnia magna and shorter response time, which could be described by the Stepwise Stress Model (SSM). Therefore movement behavior can be effectively applied in early warning of environment quality by on-line bio-monitoring. The intensive
\end{abstract}

Z. Ren $\cdot$ J. Zha $\cdot$ M. Ma $\cdot$ Z. Wang $(\bowtie)$

State Key Laboratory of Environmental Aquatic Chemistry,

Research Center for Eco-Environmental Science,

Chinese Academy of Science,

P.O. Box 2871, Beijing 100085,

People's Republic of China

e-mail: wangzj@rcees.ac.cn

A. Gerhardt

LimCo International, An der Aa 5,

D-49477 Ibbenbüren, Germany changes in behavior strength of Daphnia magna over a short time follow the SSM concept and can be used as an indicator of early stress response to OP accidental contamination.

Keywords Organophosphorous pesticide . Daphnia magna On-line bio-monitoring · Behavior strength · Stepwise Stress Model . Aquatic environmental accidents

\section{Introduction}

Organophosphorous pesticides (OPs) are among the most potent insecticides known and have been used throughout the world to control pests in agricultural crops, forests, and wetlands for more than four decades (Smith 1987). Their wide use is due to high toxicity and rapid environmental degradation (Eto 1974). Unfortunately, OPs lack target specificity and can cause severe and persistent population effects on aquatic non-target species, particularly invertebrates (Fulton and Key 2001; Schulz and Liess 1999). Extensive use of OPs and the release into aquatic environments may alter the balance of biological communities.

OPs can be taken up by organisms through the skin, the respiratory system and the digestive tract. They then react with acetyl cholinesterase (AChE) to inhibit the activity of the enzyme, which may induce 
the loss of the nerve conduction ability (Christopher et al. 2001). In invertebrates, AChE inhibitors are known to exert their toxicity on the central nervous system (Eldefrawi 1985). AChE inhibition may cause hyperactivity, loss of coordination, convulsions, paralysis and other kinds of behavioral changes (Kuhr and Dorough 1976). Hence, it may be appropriate to assess OP toxicological impact by detecting the behavioral changes in a non-target invertebrate species.

Daphnia magna, a small planktonic invertebrate crustacean $(0.5-5.0 \mathrm{~mm})$ with a short life cycle, is very sensitive to changes of the chemical composition in aquatic environments. Daphnia magna is a standard organism for toxicity tests, and much literature exists on its response to different types of toxins (Gerhardt et al. 2005; Knops et al. 2001; Peters and De Bernardi 1987). The species has often been used in bioassays and environmental monitoring of aquatic systems due to the ease and economy of maintaining cultures (Tomasik and Warren 1996). Some studies showed that Daphnia magna was very sensitive to ethyl parathion (Sturm and Hansen 1999), as it often inhabits small water bodies in and around agricultural fields receiving OP treatments (Peters and De Bernardi 1987). In addition, the movement patterns of Daphnia magna have many different components.

Stress is not something to be avoided, but the nonspecific response of an organism to any demand made upon it. A lower limit or "stress threshold" may be defined. Stress, including stress due to toxicants, will initially evoke an "alarm reaction" but no organism can maintain a chronic state of alarm and it will eventually enter a phase of adaptation or resistance (Selye 1973). The external manifestation of stress typically involves physiological responses mediated by chemical messengers and the nervous system. An organism may respond to a stressor in many ways, including changes in movement patterns.

Motility is a characteristic feature of many organisms that cannot be neglected as an important physiological factor in survival. Changes in the movement behavior of organisms can therefore be used as a suitable indicator in ecotoxicological risk assessment (Harald and Donat-P. 2001). Behavioral change in organisms associated with stress and toxicant exposure provides novel information that cannot be gained from traditional toxicological methods, which include shortterm and median lethal exposure effects, and the potential for mortality (Andrew et al. 2004). From the 1980s forward, a great deal of research on fish movements has been done to evaluate environmental stress (Park et al. 2005; Steinberg et al. 1995; Yachida et al. 1981). There are also some studies on the consequences of perinatal exposure to OPs on invertebrate behavior (Arthur et al. 1983; Roast et al. 2000). The progress of behavioral toxicology as a consensus-based discipline has been hindered by a lack of test standards, homogeneity of samples, variation in measured endpoints, and small sample sizes. Therefore, no generally accepted model for movement based on behavioral change under OP stress has yet been produced.

Though behavioral responses of Daphnia magna and other aquatic organisms to some environmental contaminants have already been investigated (Andrew et al. 2004; Harald and Donap-P. 1999; Hubert et al. 2003), hardly any literature is currently available which deals with behavior strength changes of aquatic invertebrates under contaminant stress.

In the present studies, bioassays were carried out to assess the acute effects of three model OPs: Dipterex $\left(\mathrm{C}_{4} \mathrm{H}_{8} \mathrm{Cl}_{3} \mathrm{O}_{4} \mathrm{P}\right)$, malathion $\left(\mathrm{C}_{10} \mathrm{H}_{19} \mathrm{O}_{6} \mathrm{PS}_{2}\right)$ and parathion $\left(\mathrm{C}_{10} \mathrm{H}_{14} \mathrm{O}_{5} \mathrm{NSP}\right)$ on behavior strength of Daphnia magna. The effects of the OPs on the movement behavior of Daphnia magna were observed using the movement signal detected by a Multispecies Freshwater Biomonitor ${ }^{\circledR}$ (MFB, Limico International, Germany). Finally, a behavioral change model for Daphnia magna was constructed as an early warning system of aquatic OP contamination.

\section{Materials and methods}

\section{Daphnia magna culture}

The experimental Daphnia magna (24-h young) were obtained from the Chinese Environmental Science Academy and cultured in our laboratory for more than three generations.

The culture was maintained at $20 \pm 2^{\circ} \mathrm{C}$ under a 16-h light: 8 -h dark photoperiod (illumination ranged between 3,000 and $4500 \mathrm{~lx}$ ). Culture medium was prepared according to the components of the Standard Reference Water (SRW) (ISO 1996a) and Daphnia magna were fed Scenedesmus obliquus.

Before the exposure experiments, the gravid female Daphnia magna were taken out and cultured individually in 50-ml glass beakers of SRW until they 
oviposited. As Daphnia magna neonates from the first brood are usually the smallest (Lampert 1993) and much more sensitive to different growth media, healthy neonates (about $24 \mathrm{~h}$ ) were always taken from the second and following clutches as recommended (APHA 2001). During the individual culture of female Daphnia magna with eggs, the growth medium was renewed regularly and Daphnia magna were fed Scenedesmus obliquus algae three times a week. Before feeding Daphnia magna, the culture medium of Scenedesmus obliquus was filtered and then diluted by SRW until the concentration reached $1 \times 10^{5}$ cells $/ \mathrm{ml}$. The quantity of the algae was about $1 \%$ beaker volume.

Sample preparation

Dipterex (GSGB23022-92, $100 \mathrm{mg} / \mathrm{ml} \times 1 \mathrm{ml}$ ), malathion (GSGB23018-92, $100 \mathrm{mg} / \mathrm{ml} \times 1 \mathrm{ml}$ ) and parathion (GSGB23009-92, $100 \mathrm{mg} / \mathrm{ml} \times 1 \mathrm{ml}$ ) were purchased from the Standard Sample Center of People's Republic of China.

Dimethyl sulfoxide (DMSO, analytical pure) was compatible with earlier ecotoxicity assessments (De la Torre et al. 1995), so the solvents of the three samples were added to $1 \mathrm{ml}$ DMSO. The samples were stored at $4^{\circ} \mathrm{C}$ until use.

The control concentration of DMSO was less than $0.5 \%$ in the tests. Studies showed that DMSO of such concentration would neither lead to acute toxicity to Daphnia magna nor affect the mobility of Daphnia magna (Sandbacka et al. 2000).

Acute toxicity tests

Preliminary acute toxicity tests were conducted in order to evaluate the effects of different OPs on the movement behavior of Daphnia magna. The acute test was performed in accordance with the standard protocol for Daphnia magna acute test (ISO 1996b). 24-h and 48-h static exposure experiments were performed to assess the environmental risk of the three model OPs. During the studies, laboratory conditions were kept the same as in the culturing room. All determinations were repeated three times.The evaluation of median lethal concentrations $\left(\mathrm{LC}_{50-24}\right.$ and $\left.\mathrm{LC}_{50-48}\right)$ of dipterex, malathion and parathion to Daphnia magna was regarded as an elementary step before studying the median lethal and sublethal behavioral changes under OP stress. During the exposure experiments, no food was added. Death of Daphnia magna was defined according to Villegas-Navarro et al. (1999) as the inability to swim for more than a few strokes within $15 \mathrm{~s}$ after gentle agitation of the test vessel.

Behavioral change analysis

Multispecies Freshwater Biomonitor ${ }^{\circledR}$ (MFB) is an online instrument for continuous water control. The MFB can be used as an early warning system by detecting changes in behavior of selected bioindicators to demonstrate degradation of the water quality. The measurement is based on an easy and robust measurement technique, allowing for the use of selected animal species up to 96 measurement channels (Gerhardt et al. 2002). The biosensor of MFB is located in the test chamber. There are two pairs of electrodes at the walls of each test chamber. One pair sends a high frequency signal of alternating current, which is then received by the second pair of non currentcarrying electrodes. The sending electrodes work in synchrony and hence avoid interferences between the channels. Movements of the test animals cause changes in the amplitude of the generated sinus signal, dependent on both the degree of activity of the animal and the size of the animal relative to the size of the chamber. Translated by discrete Fast Fourier Transform (FFT) designed in MFB, movement frequency signal was changed into time series data simultaneously. It provided a histrogramme of different frequencies per signal, where the height of the bars indicated how much time the animal spent on each frequency.

In situ long-term operation of MFB was carried out to obtain the behavioral change data. The monitoring data were analyzed using functions of "alarm generation", which is based on the alarm algorithm in an ARIMA-model, of MFB software package. The average values of every six long-term monitoring data records for $48 \mathrm{~h}$ exposure and every three data records for $24 \mathrm{~h}$ exposure experiments were obtained automatically to calculate the motility of tested organisms, and the data were recorded as continuous number signal transformed by FFT.

Groups of experimental Daphnia magna, each consisting of 10 individuals, were selected at random and placed in the smaller test chamber (Biosensor, 
Fig. 1 The acute toxicity of dipterex, malathion and parathion to Daphnia magna $*: p<0.05$
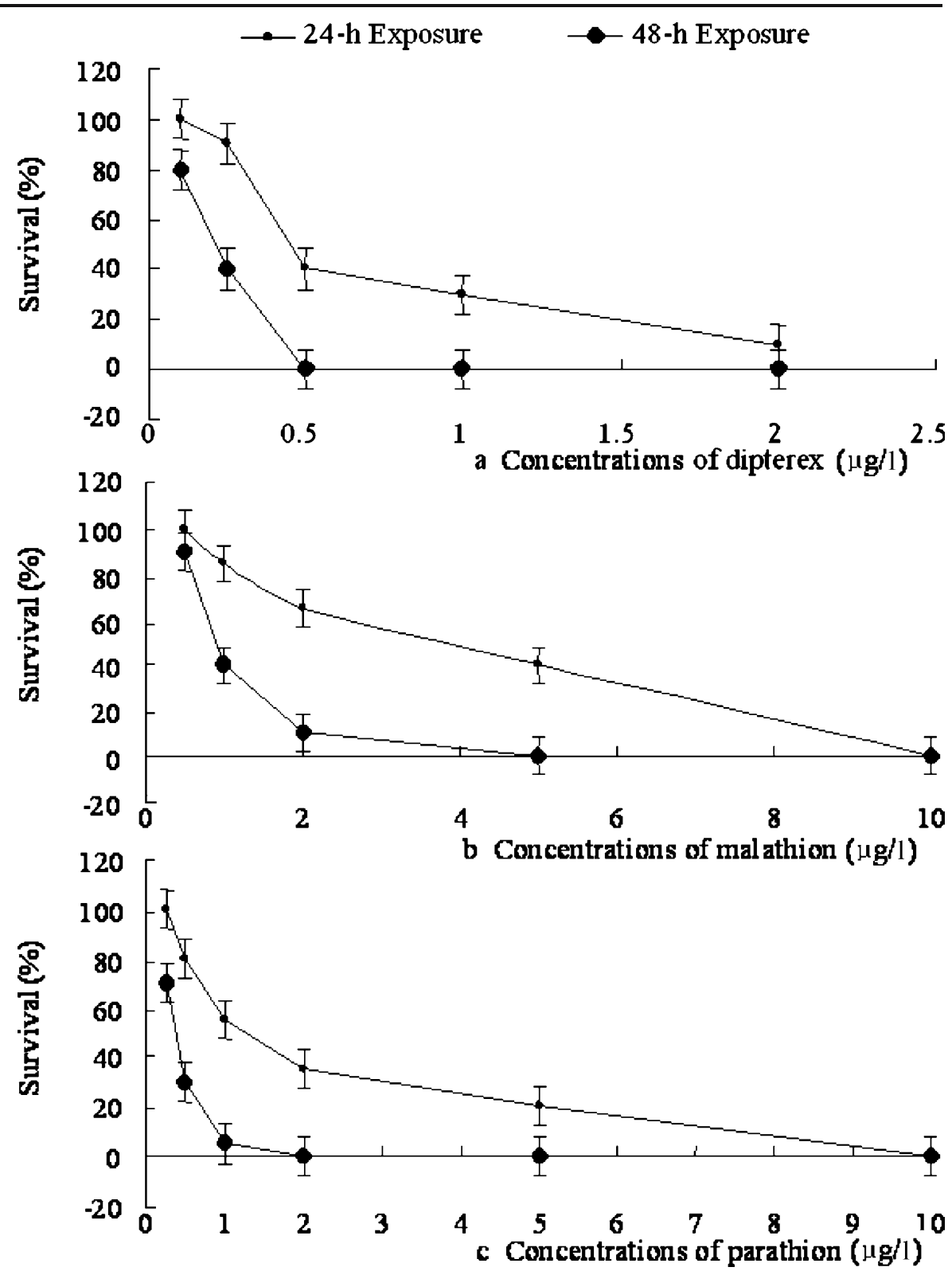

$3 \mathrm{~cm}$ long, $2 \mathrm{~cm}$ in diameter) of the MFB, which was closed off on both sides with nylon nets $(250 \mu \mathrm{m})$. The behavioral change studies were carried out in an airtight aquarium in order to decrease the effects of in vitro environmental factors on the movement behavior of Daphnia magna. No food was added during the experiments.

A 48-h exposure experiment was carried out to evaluate the effects of different OPs on the movement behavior of Daphnia magna detected by the MFB. The concentration settings of OPs were based on the results of acute toxicity tests.
In this study, behavior strength, as the main behavior parameter of Daphnia magna, was determined by changes in different frequency movements during a 48-h exposure period.

Behavior strength is one of the behavior parameters of organisms with motility. It is not a specific aspect, but an integrative reflection of all instantaneous motions, e.g. swimming velocity, behavioral frequency, and movement extent and so on. In this study, full behavior strength was regarded as 1 unit. Using the MFB, one can obtain the change of behavior strength over time, and from the change, the early warning of 
Table 1 Daphnia magna acute test: $\mathrm{LC}_{50}$ values $(\mu \mathrm{g} / \mathrm{l})$ for each organophosphorous pesticides

\begin{tabular}{llll}
\hline $\begin{array}{l}\text { Organophosphorous } \\
\text { pesticides }\end{array}$ & $\begin{array}{l}\text { Concentration } \\
\text { levels }\end{array}$ & $\begin{array}{l}\mathrm{LC}_{50-} \\
24^{*}\end{array}$ & $\begin{array}{l}\mathrm{LC}_{50-} \\
48^{*}\end{array}$ \\
\hline $\begin{array}{l}\text { Dipterex } \\
\text { Malathion }\end{array}$ & $0.10-5.00$ & 0.45 & 0.21 \\
Parathion & $0.50-10.00$ & 3.80 & 0.90 \\
& $0.25-10.00$ & 1.25 & 0.38 \\
\hline
\end{tabular}

*mean value of $\mathrm{LC}_{50}$ and with $95 \%$ confidence limit

environmental contaminants could be made. All the studies on behavioral changes were repeated three times.

In order to further demonstrate the relationship between behavior strength change and the OP concentration in water bodies, additional experiments were conducted using OP concentrations based on the existing environmental quality standard for surface water in People's Republic of China (GB3838-2002 of China P. R. 2002).

\section{Statistical analysis}

For the purpose of this study, all of the statistical data was treated by SPSS 13.0 using a statistical criterion of $p<0.05$. In this study, the average values are presented. Probit analyses using SPSS were performed to determine the acute toxicity of dipterex, malathion and parathion to Daphnia magna (LC50 for each OP). One-way analysis of variance (ANOVA) was performed to compare the effects of median lethal OP stress on the behavior strength of Daphnia magna with the controls.

\section{Results}

Acute toxicity tests

In 24-h and 48-h acute toxicity tests with neonates, the mortalities of Daphnia magna in different water bodies were first measured to evaluate $\mathrm{LC}_{50}$. Control groups were tested in a solvent solution (SRW99.5\%+ $0.5 \%$ DMSO). The measured concentration levels and the survival of Daphnia magna are shown in Fig. 1, and the calculated $\mathrm{LC}_{50}-24 \mathrm{~h}$ and $\mathrm{LC}_{50}-48 \mathrm{~h}$ values with linear interpolation are shown in Table 1 . The slopes of the probit regression lines were uniform, suggesting that the concentration-response relationship was similar for different OPs. 
Fig. 2 The effects of sublethal dipterex, malathion and parathion on the behavioral changes of Daphnia magna *: $p<0.05$

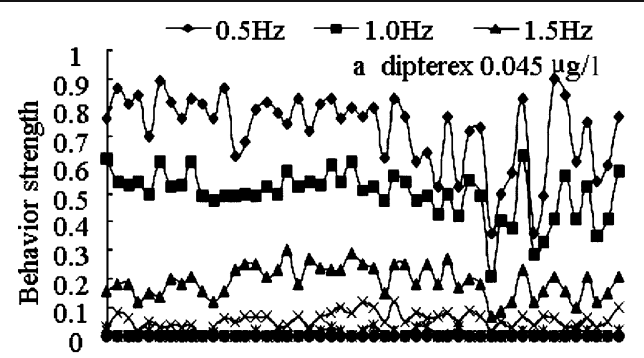

$-\times-2.0 \mathrm{~Hz}$

$\approx 2.5 \mathrm{~Hz} \quad-3.0 \mathrm{~Hz}$

b malathion $0.38 \mu \mathrm{g} / 1$
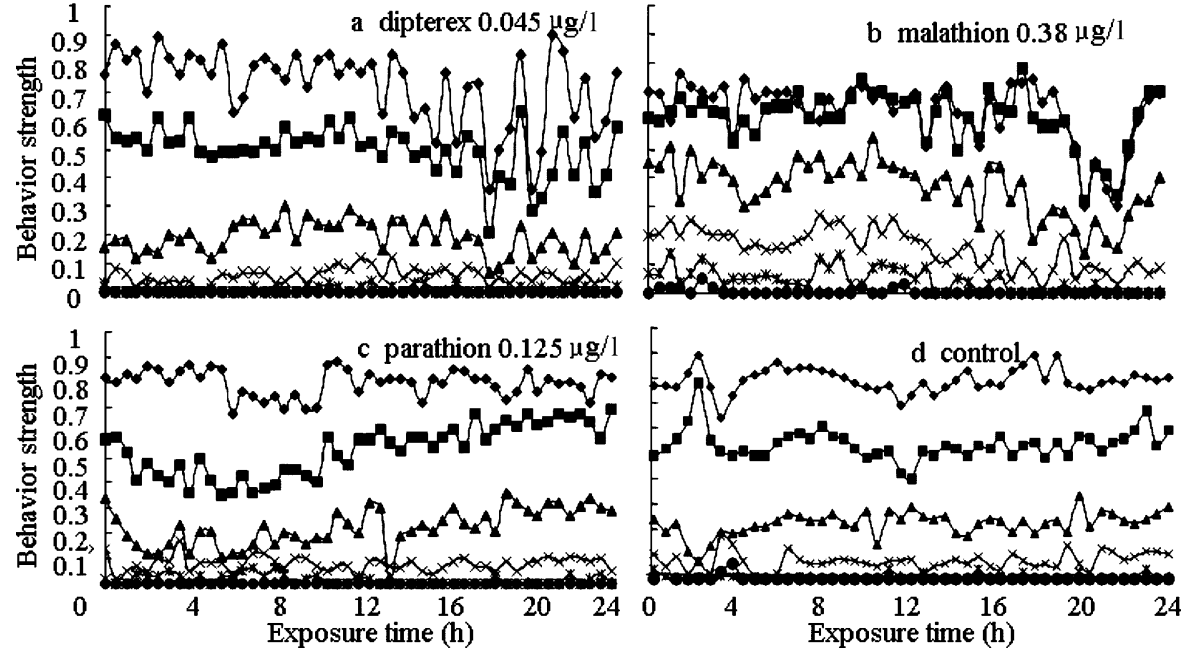

Behavioral change analysis

Sublethal concentrations may be regarded as $10 \%$ LC $_{50}$ or less (Kong et al. 2000). For the behavioral experiments, therefore, the 24-h sublethal concentrations were defined as the lower $95 \%$ confidence limit of the 24-h $\mathrm{LC}_{50}$. The sublethal exposure concentrations were $0.045 \mu \mathrm{g} / \mathrm{l}$ for dipterex, $0.38 \mu \mathrm{g} / \mathrm{l}$ for malathion and $0.125 \mu \mathrm{g} / \mathrm{l}$ for parathion. Within the lethal exposure range, concentrations of the three OPs (Dipterex: $0.45 \mu \mathrm{g} / \mathrm{l}, 0.21 \mu \mathrm{g} / \mathrm{l}$; Malathion: $3.80 \mu \mathrm{g} / \mathrm{l}$, $0.90 \mu \mathrm{g} / \mathrm{l}$; Parathion: $1.25 \mu \mathrm{g} / \mathrm{l}, 0.38 \mu \mathrm{g} / \mathrm{l}$ ) were selected to carry out the behavioral change studies on Daphnia magna under median lethal stress. SRW99.5\%+0.5\% DMSO was used as the control.

Analyzed by FFT, the movement frequencies were most often measured at $0.5,1.0,1.5,2.0,2.5$ and $3.0 \mathrm{~Hz}$, with less than $0.1 \%$ of movement behavior exceeding $3.0 \mathrm{~Hz}$ (Table 2). Therefore, movement behavior between $0.5 \mathrm{~Hz}$ and $3.0 \mathrm{~Hz}$ was chosen to indicate behavior strength changes under OP stress.

In this study, movement behavior data were taken once an hour for $48 \mathrm{~h}$ exposure and twice an hour for $24 \mathrm{~h}$ exposure. The continuous number signal translated by FFT in MFB could be exported in the form of Excel worksheet. In movement behavior data processing, the average values are discussed. At first, the synchronous monitoring data of the same movement frequency of three replications for each treatment were averaged, and then the averages of different movement frequencies were used to evaluate the effects of different concentrations OPs on the movement behavior of Daphnia magna.

The behavioral responses of Daphnia magna to sublethal and median lethal OP stress with $p<0.05$ are shown in Figs. 2 and 3. Within the control group, no significant changes in behavior were detected. In the case of OP exposures, the results demonstrate that the changes of movement behavior coincided at the different frequencies for the different OPs; that is, movements of the same frequency changed to the same extent. The changes of behavior strength depended mainly on the lower frequency movements $(0.5 \mathrm{~Hz}$ and $1.0 \mathrm{~Hz})$. And, in general, the relationship between behavior strength and movement frequency was inverse: the higher the movement frequency, the lower the behavior strength. Behavioral changes showed an evident concentration-response relationship with median lethal OP stress (Table 2). The movement behavior of different frequencies changed simultaneously, and higher concentrations of dipterex, malathion and parathion resulted in a greater degree of the behavioral changes, as well as a shorter response time for behavioral changes.

The changing trends of behavior strength to median lethal OP stress were the same as in higher concentrations designed according to environmental quality standard for surface water in People's Republic of China (GB3838-2002 of China P. R. 2002) (Fig. 4), with the exception of a shorter response time. Even under the sublethal stress, the behavioral strength changes of Daphnia magna were evident (Fig. 2), 
Fig. 3 The effects of median lethal dipterex, malathion and parathion on the behavioral changes of Daphnia magna *: $p<0.05$

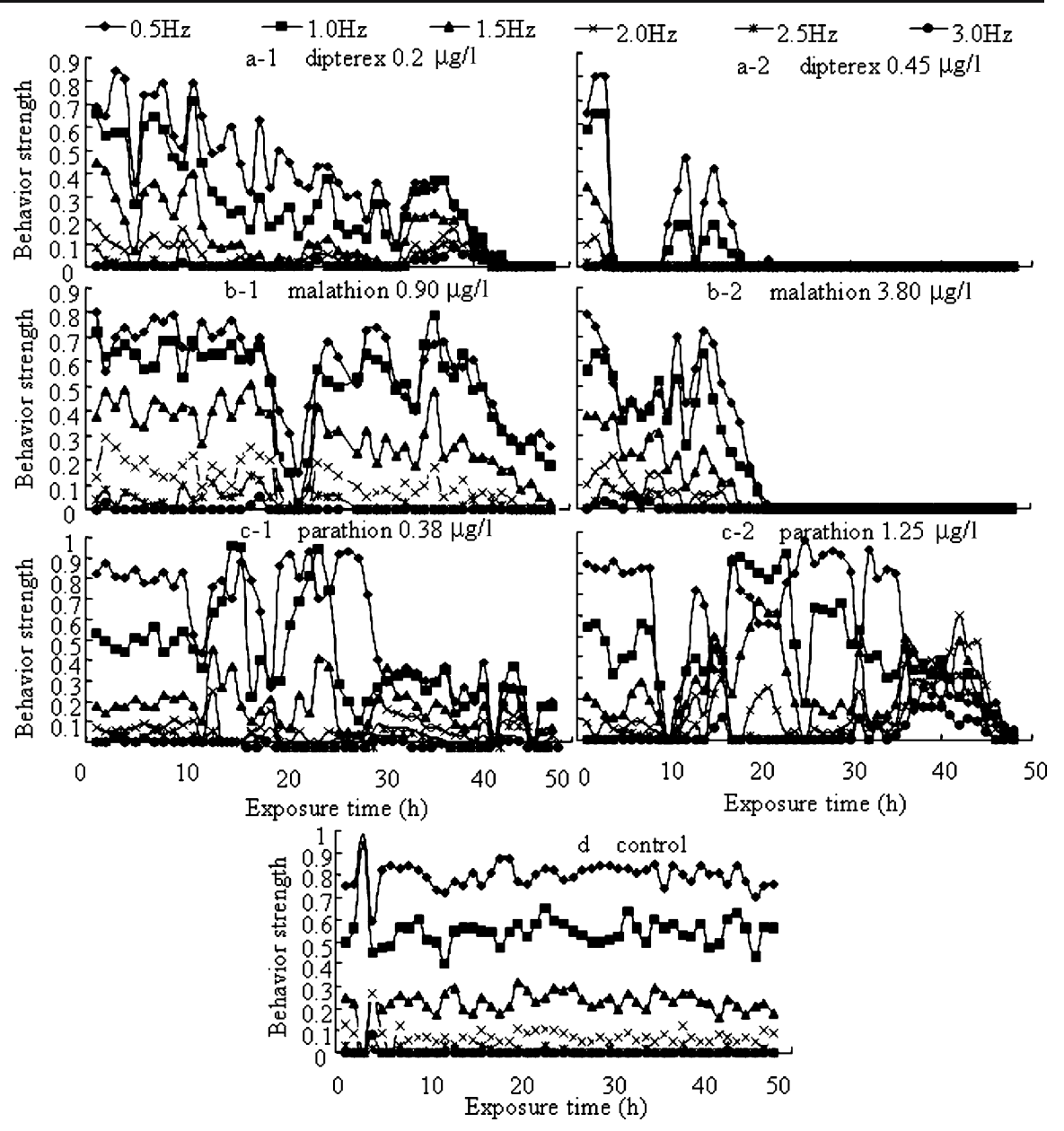

which showed that the behavior strength was more sensitive in monitoring aquatic environment quality changes than the other parameters in acute toxicity.

\section{Discussion}

Acute toxicity tests

The toxic action of OPs is similar (Christopher et al. 2001; Eldefrawi 1985; Kuhr and Dorough 1976) in that they inhibit AChE leading to the loss of nerve conduction ability (Eldefrawi 1985). Tables 1 and 2 demonstrate that the three OPs were highly toxic to Daphnia magna and that the acute toxicity sequence was dipterex $>$ parathion $>$ malathion. Other invertebrates and some fish also demonstrated the same order of toxicity among the three OPs, including fresh water teleost Saccobranchus fossilis (Verma et al.
1982), midges (Belden and Lydy 2001), grass shrimp Palaemonetes pugio (Peter et al. 1998) and goldfish Carassius auratus (Ana et al. 2004). This suggested that the acute relative toxicity of the OPs might be analogous across these species.

Behavioral change analysis

The results of acute toxicity and the behavioral studies showed that behavioral changes were timedependent. There were also obvious concentrationresponse relationships between the three model OPs and the behavioral response of Daphnia magna. Behavioral response of Daphnia magna to sublethal and median lethal OP stress (Figs. 2 and 3) suggested that whether exposed in high or low concentrations OPs, the movements of Daphnia magna changed similarly. Additionally, the strength of movement behavior detected at different frequencies showed a 
Fig. 4 The effects of higher concentrations dipterex, malathion and parathion on the behavioral changes of Daphnia magna *: $p<0.05$
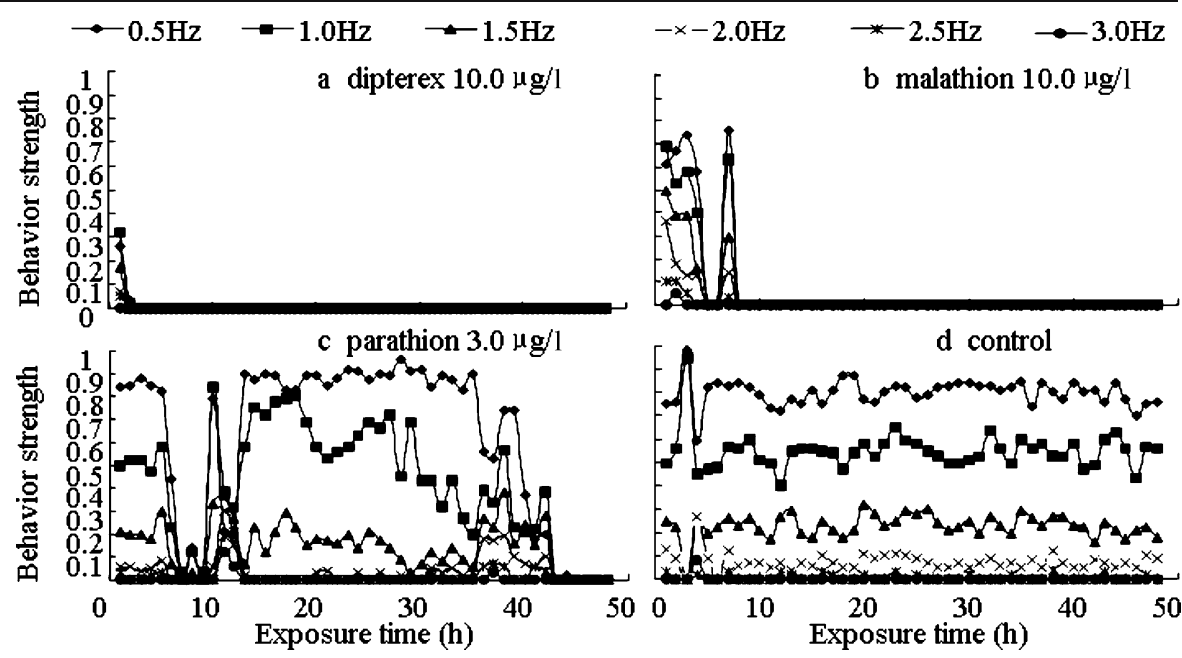

sudden decrease after a certain length of exposure, although after a period of low strength time, motility resumed. At the same time, the readjusted behavior strength showed some differences:

(1) In dipterex exposure, the readjusted behavior strength of different frequencies changed simultaneously. It was obviously lower than the first exposure period and a gradual decreasing trend occurred, e.g. a-1 and a-2 in Fig. 3;

(2) In parathion exposure, the discrepancy between the adjusted behavior strength and start motility was not clear, but after a period of maintenance, there was a sudden decrease to immobility, e.g. b-1 and b-2 in Fig. 3;

(3) In malathion exposure, after motility resumed, the behavioral changes showed greater diversity among different frequency movements. Different frequency movements were displayed separately. Behavioral strength sometimes decreased or increased suddenly. After a period of maintenance, a second depression occurred, and occasionally, motility resumed afterward, e.g. c-1 and c-2 in Fig. 3. These changes were different from the regular ones in other exposures (a-1, a-2, b-1 and b-2 in Fig. 3).

(4) The behavioral response trends of Daphnia magna to sublehtal OP stress (Fig. 2) were similar at median lethal concentrations and the degree of change showed significant concentration-response relationships.

The movements of Daphnia magna changed more significantly at higher concentrations than under median lethal OP stress (Fig. 4). This result suggested that the movement behavior of Daphnia magna could be feasible as an early warning system for aquatic environmental quality by on-line monitoring behavior strength changes, according to the requirements of the environmental quality standard for surface water of People's Republic of China.

Stepwise Stress Model (SSM) under OP stress

The Stepwise Stress Model (SSM), described by Gerhardt (Gerhardt 1999, 2001; Gerhardt et al. 2005, anticipates that with either increasing toxicant concentration or exposure time, a cascade of regulatory behavioral stress responses are activated and performed by the organisms. SSM postulates that an organism displays a time-dependent sequence of different regulatory or compensatory behavioral stress responses during exposure to pollutants above their respective thresholds of resistance. An increasing stress stimulus provokes regulatory responses (loading stress). Above a certain stimulus level, however, several reactions are possible: (1) the homeostasis cannot be maintained and a toxic effect in

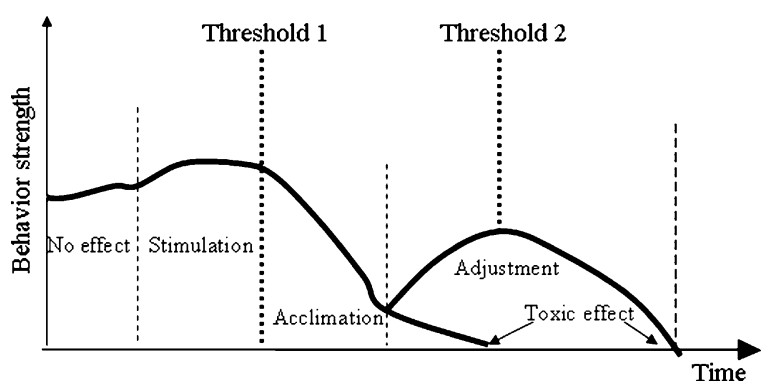

Fig. 5 Stepwise Stress Model (SSM) under OP stress 
the organism occurs (limiting stress), (2) the organism can acclimate to the increased stress level and (3) the organism decreases the performance of the response and increases the performance of another response to the stimulus. If the first stress response decreases to less than the original level, a toxic effect occurs.

According to the relationship between the exposure time and the movement behavioral changes of Daphnia magna in each kind of OP, along with the behavioral change trends shown in Figs. 2, 3 and 4, we found that the behavioral change trends of Daphnia magna were consistent with SSM and proved SSM for the first time for OPs in Daphnia magna, by showing changes in behavioral strength (movements characterized by different signal frequencies). Based on the reports of Gerhardt in 1999 and 2001, the theoretical reactions of Daphnia magna in a certain behavior under OP stress that included the categories No effect, Stimulation, Acclimation, Adjustment, and Toxic effect are shown in Fig. 5.

In theory, the first behavior modulation of Daphnia magna was to increase the strength of all frequency movements presumably to try to escape from the polluted aquatic environment (Avoidance behavior), which explains the behavioral changes observed in Stimulation after No effect. Presumably, certain OP concentrations would be too high for Daphnia magna to start Avoidance behavior because this type of behavior was absent or of short duration at high OP concentrations (Fig. 4). In these cases, behavior strength tended to decrease gradually until the ability for movement was lost. In other cases, Daphnia magna were apparently able to cope with the neurotoxic stressors and increased behavioral strength. Associated with this response, the length of Stimulation was close to the expected value, about $1-3 \mathrm{~h}$ (Fig. 3) and it was in inverse ratio to the OP concentrations.

Stimulation was usually followed by Acclimation which was a continuous weakening of behavior strength. The main reason for the fall of movement behavior in Acclimation was that the behavior adjustment reached extreme of "Alarm reaction" (Threshold 1). As time passed, stress gradually decreased the motility of Daphnia magna.

After Acclimation, movement behavior split in two directions: First, if Daphnia magna could not overcome Threshold 1, Toxic effect would occur. Secondly, if Daphnia magna lived through Threshold 1, the movement behavior strength resumed in a short time as shown in Adjustment and there would be a second extreme of "Alarm reaction" (Threshold 2) until Toxic effect occurred.

Based on these results, "avoidance behavior" is quite appropriate to be used in early warning pollution stress responses. Almost all aquatic animals have the ability of actively escaping from a polluted environment to an unpolluted area (Robin and Don 1971). A trend for behavior modulation to maintain a stable internal environment and diminish dependence on an external environment was also shown (Putman and Wratten 1984).

\section{Conclusions}

This study proved behavioral responses of the freshwater invertebrate cladoceran Daphnia magna to be fast and sensitive. Median lethal and sublethal bio-indicators to OP stress and the movement behavior under OP stress showed regular changes. Movement behavior can be effectively applied in early warning of environment quality by on-line bio-monitoring. The intensive changes in "behavior strength" of Daphnia magna over a short time follow the SSM concept and can be used as an indicator of early stress response to OP contamination. This knowledge could be applied to monitor aquatic environmental accidents. If in the future, relationships between median lethal contaminant stress and behavior responses could be described by a mathematical formula, early warning systems would be greatly improved. To this aim, more studies regarding the behavioral changes of invertebrates under different types of contaminant stress are needed to further prove the SSM.

Acknowledgements We sincerely thank Professor Philip A. Spear, Centre de recherche TOXEN, Université du Québec à Montréal, for revising the English. This work was supported by Natural Science Foundation of China (40471129), Natural Science Foundation of Beijing Municipality (8061004) and Chinese Academy of Sciences (KZCX3-SW-431).

\section{References}

Ana, F., Andres, V., \& Ana, M. (2004). Time course of brain cholinesterase inhibition and recovery following acute and subacute azinphosmethyl, parathion and carbaryl exposure in the goldfish (Carassius auratus). Ecotoxicology and Environmental Safety, 57(3), 420-425.

Andrew, S. K., James, D. S., Geoffrey, T. G., Timothy, C.A. M., \& Colin, H. (2004). A video-based movement analysis system to quantify behavioral stress responses of fish. Water Research, 38, 3993-4001. 
APHA (2001). Standard methods for the examination of water and wastewater (20th ed.). American Public Health Association, American Water Works Association, and Water Pollution Control Federation, Washington, DC, 20005.

Arthur, J. W., Zischke, J. A., Allen, K. N., \& Hermanutz, R. O. (1983). Effects of diazinon on macroinvertebrates and insect emergence in outdoor experimental channels. Aquatic Toxicology, 4, 283-302.

Belden, J. B., \& Lydy, M. J. (2001). Effects of atrazine on acetylcholinesterase activity in midges (Chironomus tentans) exposed to organophosphorus insecticides. Chemosphere, 44 (8), 1685-1689.

Christopher, M. T., David, J. S., \& Matthew, J. W. (2001). Fluorinated phosphorus compounds: Part 4. A lack of anticholinesterase activity for four tris (fluoroalkyl) phosphates. Journal of Fluorine Chemistry, 107(1), 155-158.

De la Torre, A. I., Fernández, C., Tarazona, J. V., \& Muñoz, M. J. (1995). Detection of aroclor, DDT, malathion and HCB using semipermeable membranes as concentration method. Chemosphere, 31(2), 2727-2737.

Eldefrawi, A. T. (1985). Comparative insect physiology, biochemistry, and pharmacology (pp. 115-130). Oxford: Pergamon.

Eto, M. (1974). Organophosphorous pesticides: Organic and biological chemistry. Ohio: CRC.

Fulton, M. H., \& Key, P. B. (2001). Acetylcholinesterase inhibition in esturaine fish and invertebrates as an indicator of organophosphorus insecticide exposure and effects. Environmental Toxicology and Chemistry, 20, 37-45.

GB3838-2002 of China P. R. (2002). Environmental quality standard for surface water. China: Environmental Science.

Gerhardt, A. (1999). Recent trends in on-line biomonitoring for water quality control. In A. Gerhardt (Ed.), Biomonitoring of polluted water. Reviews onactual topics. Environmental Research Forum 9. Zurich, Switzerland: Trans Tech.

Gerhardt, A. (2001). A new multispecies freshwater biomonitor for ecological relevant control of surface waters. In F. Butterworth, A. Gunatilaka, \& M. E. Gonsebatt (Eds.), Biomonitorsand biomarkers as indicators of environmental change. Environmental Science Forum 56; vol. 2. New York: Kluwer-Plenum.

Gerhardt, A., Janssens de Bisthoven, L., Mo, Z., Wang, C., Yang, M., \& Wang, Z. (2002). Short-term responses of Oryzias latipes (Pisces: Adrianichthyidae) and Macrobrachium nipponense (Crustacea: Palaemonidae) to municipal and pharmaceutical wastewater in Beijing, China: Survival, behavior, biochemical biomarkers. Chemosphere,47, 35-47.

Gerhardt, A., Janssens de Bisthoven, L., \& Soares, A. M. V. M. (2005). Evidence for the Stepwise Stress Model: Gambusia holbrooki and Daphnia magna under AMD and ACID stress. Environmental Science \& Technology, 39(11), 4150-4158.

Harald, T., \& Donap-P., H. (1999). Fast examination of water quality using the automatic biotest ecotox based on the movement behavior of a freshwater flagellate. Water Research, 33(2), 426-432.

Harald, T., \& Donat-P., H. (2001). Automated biomonitoring using real time movement analysis of Euglena gracilis. Ecotoxicology and Environmental Safety, 48, 161-169.

Hubert, U., Jördis, K., \& Helmut, K. (2003). Behavioural response of the cladoceran Daphnia magna Straus to sublethal Copper stress-validation by image analysis. Aquatic Toxicology, 65, 435-442.
ISO (1996a). Water Quality-determination of the acute lethal toxicity of substances to a freshwater fish [Brachdanio rerio (Hamilton-Buchanan), Teleostei, Cyprinidae] - Part 3: Flow-through method. ISO/ DIS 7346/3.

ISO (1996b). Water quality - Determination of the mobility of Daphnia magna Straus (Cladocera, Crustacea). ISO 6341. Geneva, Switzerland.

Knops, M., Altenburger, R., \& Segner, H. (2001). Alterations of physiological energetics, growth and reproduction of Daphnia magna under toxicant stress. Aquatic Toxicology, 53, 79-90.

Kong, F., Yin, D., \& Yan, G. (2000). Environmental biology. Beijing: Higher Education (in Chinese).

Kuhr, R. J., \& Dorough, H. W. (1976). Mode of action. In Carbamate insecticides: Chemistry, biochemistry, and toxicology (pp. 41-70). Cleveland, OH: CRC.

Lampert, W. (1993). Phenotypic plasticity of the size at first reproduction in Daphnia: The importance of material size. Ecology, 74, 1455-1466.

Park, Y. S., Chung, N., Choi, K. H., Cha, E. Y., Lee, S. K., \& Chon, T. S. (2005). Computational characterization of behavioral response of medaka (Oryzias latipes) treated with diazinon. Aquatic Toxicology, 71(3), 215-228.

Peters, R. H., \& De Bernardi, R. (1987). Daphnia." Memorie dell' Instituto Italiano di Idrobiologia Dott. Marco de Murchi, 45. Verbania Pallaza.

Peter, B. K., Michael, H. F., Geoffrey, I. S., Shawn, L. L., \& Edward, F. W. (1998). Lethal and sublethal effects of malathion on three life stages of the grass shrimp, Palaemonetes pugio. Aquatic Toxicology, 40(4), 311-322.

Putman, R. J., \& Wratten, S. D. (1984). Principles of ecology. London and Canberra: Groom Helm.

Roast, S. D., Widdows, J., \& Jones, M. B. (2000). Disruption of swimming in the hyperbenthic mysid Neomysis integer (Peracarida mysidacea) by the organophosphate pesticide chlorpyrifos. Aquatic Toxicology, 47, 227-241.

Robin, H., \& Don, R. A. (1971). Shrimps in relation to oxygen depletion and its ecological significance in a polluted estuary. Environmental Pollution, 2(1), 13-35.

Sandbacka, M., Christianson, I., \& Isomaa, B. (2000). The acute toxicity of surfactants on fish cells, Daphnia magna and fish A comparative study. Toxicology in vitro, 14, 61-68.

Schulz, R., \& Liess, M. (1999). A field study of the effects of agriculturally derived input on stream macroinvertebrate dynamics. Aquatic Toxicology, 46, 155-176.

Smith, G. J. (1987). Pesticide use and toxicity in relation to wildlife: Organophosphorus and carbamate compounds. Washington, DC: United States Department of the Interior, Fish and Wildlife Service, Resource.

Steinberg, C. E. W., Lorenz, R., \& Spieser, O. H. (1995). Effects of atrazine on swimming behavior of zebrafish, Brachydanio rerio. Water Research, 29, 981-985.

Sturm, A., \& Hansen, P. D. (1999). Altered cholinesterase and monooxygenase levels in Daphnia magna and Chironomus riparius exposed to environmental pollutants. Ecotoxicology and Environmental Safety, 42, 9-15.

Selye, H. (1973). The evolution of the stress concept. American Scientist, 61, 692-699.

Tomasik, P., \& Warren, D. M. (1996). The use of Daphnia in studies of metal pollution of aquatic systems. Environmental Research, 4, 25-64. 
Verma, S. R., Bansal, S. K., Gupta, A. K., Pal, N., Tyagi, A. K., Bhatnagar, M. C., et al. (1982). Bioassay trials with twenty three pesticides to a fresh water teleost, saccobranchus fossilis. Water Research, 16(5), 525-529.

Villegas-Navarro, A., Romero, G. M. C., Rosas, L. E., Dominguez, A. R., \& Sachetin, M. W. (1999). Evaluation of
Daphnia magna as an indicator of toxicity and treatment efficy of textile wastewaters. Environment International, 25 (5), 619-624.

Yachida, M., Asada, M., \& Tsuji, S. (1981). Automatic analysis of moving images. IEEE Transactions on Pattern Analysis and Machine Intelligence, 3, 12-20. 